Journal of Applied Fluid Mechanics, Vol. 15, No. 2, pp. 427-440, 2022.

Available online at www.jafmonline.net, ISSN 1735-3572, EISSN 1735-3645.

https://doi.org/10.47176/jafm.15.02.33068

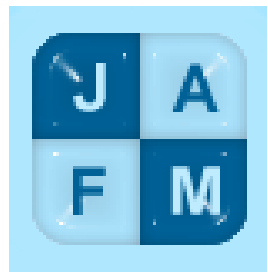

\title{
Flow Separation Control on NACA0015 Airfoil Using Synchronized Jet Actuator
}

\author{
F. Sonkaya ${ }^{1 \dagger}$, S. Cadirci $^{1}$ and D. Erdem ${ }^{2}$ \\ ${ }^{1}$ Department of Mechanical Engineering, Istanbul Technical University, Istanbul, 34437, Turkey \\ ${ }^{2}$ Department of Aeronautical Engineering, Istanbul Technical University, Istanbul, 34469, Turkey
}

†Corresponding Author Email: fatihsonkaya61@gmail.com

(Received May 31, 2021; accepted September 28, 2021)

\begin{abstract}
In this study, a novel fluidic jet actuator is designed to control flow separation on a NACA0015 airfoil at various angles of attack. The U-shaped jet actuator has two rectangular slots implemented near the leading edge of the airfoil. It is driven by a piston mechanism and operates at three excitation frequencies. Depending on the motion of the mechanism, a synchronized jet flow is generated by blowing and suction at the dual exits of the actuator slots. The experimental studies are carried out in a subsonic wind tunnel. The unsteady 2D Computational Fluid Dynamics simulations are performed by Detached Eddy Simulation with the SST k- $\omega$ turbulence model where measured jet velocities at the exits of the actuator slots are imposed as boundary conditions to mimic motion of the piston. The results at the on-mode and off-mode of the actuator are evaluated in terms of surface pressure coefficient distributions on the airfoil and averaged aerodynamic force coefficients. At low angles of attack, there is an adequate match between numerical and experimental results for the base flow without any control. At higher angles of attack, flow separation becomes considerably dominant and stall prevention by active flow control is detected especially at high excitation frequencies.
\end{abstract}

Keywords: Active flow control; Flow separation control; Jet actuator; Detached eddy simulation.

\section{NOMENCLATURE}

$\begin{array}{lll}A_{j e t} & \text { maximum jet velocity at the exit } & R_{\text {jet }} \\ C & \text { chord length } & t \\ C_{D} & \text { drag coefficient } & U_{\infty} \\ C L & \text { lift coefficient } & U_{y} \\ & & \\ C P & \text { pressure coefficient } & U_{I} \\ C \mu & \text { momentum coefficient } & U_{2} \\ f & \text { frequency } & V_{j e t} \\ R e & \text { reynolds number } & y^{+}\end{array}$

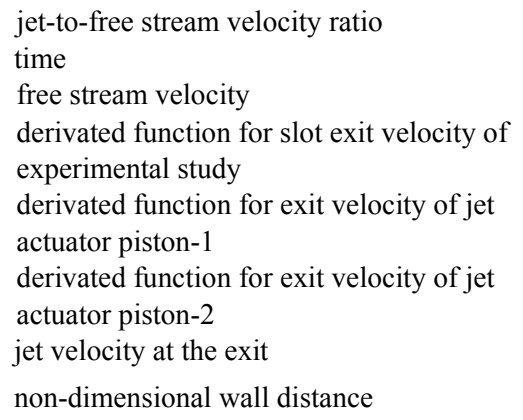

\section{INTRODUCTION}

Flow control approaches have a wide range of engineering goals such as prevention or delay of boundary layer separation, delay of transition, drag reduction and lift enhancement. Generally, flow control strategies can be classified as passive and active or a combination of both. While active flow control requires an external energy source, passive control does not require any auxiliary power (Mohamed 2001).

Active flow control studies cover a wide range of strategies using different types of actuators. Many of these applications are based on fluidic actuators which interact with the boundary layer on the airfoil surface by either suction or blowing or both (Cattafesta and Sheplak 2011). With this flow 
control strategy, it is possible to delay stall and improve aerodynamic characteristics. In many studies, the effects of jet actuators are investigated numerically and experimentally focusing mostly on the geometric parameters such as slot location, slot geometry and slot width, and actuation parameters such as jet actuator type, jet velocity, jet angle and number of jets.

Actuators acting by blowing and suction can be designed in many ways, jets can be in steady-state or they can show an unsteady behavior. Time dependent variation of suction-blowing can be generated by a sinusoidal motion and forced pulsation. Seifert et al. (1993) experimentally studied delay of boundary layer separation on a flapped NACA0015 airfoil by using oscillatory and steady blowing. They found that oscillatory blowing was more efficient compared to steady blowing. Godard et al. (2006) applied active flow control by continuous and pulsed jets emanating from crosswise located slots on an airfoil and compared their performance against passive flow control by vortex generators presented in their previous study (Godard and Stanislas 2006a). In like manner, De Giorgi et al. (2015) tested the performance of synthetic jet actuator (SJA) and continuous jet actuator (CJA) with identical momentum coefficients and identical amounts of energy fed into the cross flow. They numerically showed that the use of SJA was more advantageous than CJA if applied on a NACA0015 airfoil and compressor stator cascade. Another study on this subject is the study carried out by Genc et al. (2011). They studied the effects of different actuation parameters with and without single and simultaneous blowing and suction experimentally and numerically. Within the scope of this study, they investigated cases of steady blowing or steady suction from both slots and steady suction from one slot with steady blowing from the other slot.

Other parameters investigated in jet-induced active flow control in the literature are exit location and exit angle of jet slot. Zhao and Zhao (2014) investigated the effect of angle of the synthetic jets, jet frequency, jet momentum coefficient along with number and position of the jets on aerodynamic characteristics of the airfoil. In another study, Huang et al. (2004) carried out numerical analyses by creating jet actuators at various angles and locations on the NACA0012 airfoil. They showed and compared the effects of various amplitudes and different actuation methods. Other studies focusing on the effect of jet actuator position conducted by Müller-Vahl et al. (2015) and Buchmann et al. (2013) also contribute to the literature.

The most important parameters that affect airfoil performance in active flow control applications can be stated as $R_{j e t}, C_{\mu}$ and jet frequency. Although different coefficients are defined in various studies, it is possible to convert these coefficients to each other. Examples for studies examining the effect of such control parameters are those of Gilarranz et al. (2005a, b) and Godard and Stanislas (2006b). Gilarranz et al. (2005a,b) designed a SJA and applied it to flow separation control on NACA0015 airfoil.
They showed that increasing jet-to-free stream velocity ratio $\left(R_{j e t}\right)$ improved pressure coefficient $\left(C_{P}\right)$ distribution on the airfoil however, the effect of this enhancement decreased after a certain level. These studies provide detailed information about the design characteristics and performance of the synthetic jet actuators they have developed. On the other hand, Godard and Stanislas (2006b) conducted active flow control on an airfoil and investigated the effect of continuous jet blowing from round exits. They showed the influence of $R_{\text {jet al }}$ ong with some other actuation parameters of the round jet vortex actuator.

Although there are numerous studies in the literature on use of jet actuators on airfoil, the number of studies that provide detailed design is limited for practical applications. An example of these applications is the study by (Gilarranz et al. (2005a, b). Gilarranz et al. (2005a) first designed a synthetic jet actuator and reported the details of their design and operation limits in the experimental setup. The SJA generated sinusoidal blowing and suction and allowed manipulating $R_{\text {jet }}$ using different excitation frequencies. In a further study Gilarranz et al. (2005b) experimentally investigated the influence of their SJA design on aerodynamic characteristics of a NACA0015 airfoil under various AoA with the aim to prevent flow separation. In addition to these experimental studies, numerical studies were carried out on the same subject. One of them is the study done by Duvigneau and Visonneau (2006), in which URANS based CFD studies were performed within the scope of this study. They optimized a SJA for aerodynamic stall control by changing various parameters of SJA in order to obtain the optimum results for force characteristics and observed significant improvement in stall delay. Another numerical study based on the reference design by Gilarranz et al. (2005a, b) was done by You and Moin (2009). You and Moin (2009) performed 3D LES calculations for the same configuration reported by Gilarranz et al. (2005a, b) and validated their numerical results by these experimental data with a perfect match.

In the present study, a new jet actuator design with dual slots on a NACA0015 airfoil was developed which is similar in some fashion to the design and working principle of the SJA mentioned by Gilarranz et al. (2005a). The novelty of the present study relies on introducing a new jet actuator with multiple slots which generates blowing and suction simultaneously at an adjustable excitation frequency and operates at moderate free stream Reynolds number. To highlight the originality of the current study which is intended to contribute to the literature, related studies are summarized in Table 1.

\section{JET ACTUATOR DESIGN}

The jet actuator in the present study is developed by modifying the original jet actuator design proposed by Gillarenz et al. (Gilarranz et al. 2005a) which has a single slot near the leading edge of a NACA0015 
Table 1 Review of AFC studies with jet actuator on airfoil.

\begin{tabular}{|c|c|c|c|c|c|c|c|}
\hline Article & $\begin{array}{c}\text { AFC } \\
\text { Configuratio } \\
n\end{array}$ & $\begin{array}{c}\text { Exit } \\
\text { Location of } \\
\text { Jet Flow } \\
(\mathrm{x} / \mathrm{C}) \\
\end{array}$ & $\begin{array}{c}\text { Exit } \\
\text { Angle } \\
\text { of Jet } \\
\text { Flow }\left(^{\circ}\right) \\
\end{array}$ & $\mathrm{R}_{\text {jet }}$ & Airfoil & $\mathrm{Re}$ & Method \\
\hline $\begin{array}{c}\text { Huang et } \\
\text { al. }\end{array}$ & $\begin{array}{l}\text {-Steady } \\
\text { Suction SJ } \\
\text {-Steady } \\
\text { Blowing SJ }\end{array}$ & $0.03---0.8$ & $\begin{array}{c}-90 /- \\
30 / 0 \\
30 / 60 / 9 \\
0 \\
\end{array}$ & $\begin{array}{c}0.01- \\
0.5\end{array}$ & $\begin{array}{c}\text { NACA001 } \\
2\end{array}$ & $5 \times 10^{5}$ & Numerical \\
\hline $\begin{array}{l}\text { Genc et } \\
\text { al. }\end{array}$ & $\begin{array}{l}\text {-Steady } \\
\text { Suction SJ } \\
\text {-Steady } \\
\text { Blowing SJ } \\
\text {-Steady } \\
\text { Suction - } \\
\text { Steady } \\
\text { Blowing MJ }\end{array}$ & $\begin{array}{c}0.1 / 0.26 / 0.3 \\
6\end{array}$ & $\begin{array}{c}-90 /- \\
45 /-30 \\
30 / 45 / 9 \\
0\end{array}$ & $\begin{array}{c}0.03- \\
0.15 \\
6\end{array}$ & $\begin{array}{c}\text { NACA241 } \\
5\end{array}$ & $2 \times 10^{5}$ & $\begin{array}{c}\text { Experimenta } \\
1 \\
\text { Numerical }\end{array}$ \\
\hline $\begin{array}{c}\text { Seifert et } \\
\text { al. }\end{array}$ & $\begin{array}{l}\text {-Steady } \\
\text { Blowing SJ } \\
\text {-Oscillating } \\
\text { Blowing SJ }\end{array}$ & 0.75 & $20 / 40$ & $\begin{array}{c}0.35- \\
3.5\end{array}$ & $\begin{array}{c}\text { NACA001 } \\
5 \\
\text { (with flap) }\end{array}$ & $\begin{array}{l}1 \times 10^{5} \\
1 \times 10^{6}\end{array}$ & $\begin{array}{c}\text { Experimenta } \\
1\end{array}$ \\
\hline $\begin{array}{c}\text { Gilarranz } \\
\text { et al. }\end{array}$ & $\begin{array}{l}\text {-Sinusoidal } \\
\text { Blowing - } \\
\text { Suction SJ }\end{array}$ & 0.12 & 30 & $\begin{array}{l}1.03- \\
2.31\end{array}$ & $\begin{array}{c}\text { NACA001 } \\
5\end{array}$ & $9 \times 10^{5}$ & $\begin{array}{c}\text { Experimenta } \\
1\end{array}$ \\
\hline $\begin{array}{c}\text { Buchman } \\
\text { n et al. }\end{array}$ & $\begin{array}{l}\text {-Sinusoidal } \\
\text { Blowing - } \\
\text { Suction SJ } \\
\end{array}$ & 0 & 90 & 0.22 & $\begin{array}{c}\text { NACA001 } \\
5\end{array}$ & $3 \times 10^{4}$ & $\begin{array}{c}\text { Experimenta } \\
1\end{array}$ \\
\hline $\begin{array}{l}\text { Guoqing } \\
\text { and Qijun }\end{array}$ & $\begin{array}{l}\text {-Sinusoidal } \\
\text { Blowing - } \\
\text { Suction SJ } \\
\text {-Sinusoidal } \\
\text { Blowing - } \\
\text { Suction MJ } \\
\end{array}$ & $0.05---0.6$ & 0---90 & - & OA213 & $8.5 \times 10^{6}$ & Numerical \\
\hline $\begin{array}{l}\text { Muller- } \\
\text { Vahl et al. }\end{array}$ & $\begin{array}{l}\text {-Steady } \\
\text { Blowing SJ }\end{array}$ & $0.05 / 0.5$ & 20 & $\begin{array}{l}0.65- \\
2.65\end{array}$ & $\begin{array}{c}\text { NACA001 } \\
8\end{array}$ & $\begin{array}{c}1.25 \times 10 \\
5 \\
3.75 \times 10 \\
5\end{array}$ & $\begin{array}{c}\text { Experimenta } \\
1\end{array}$ \\
\hline $\begin{array}{l}\text { Present } \\
\text { Study }\end{array}$ & $\begin{array}{l}\text { - } \\
\text { Synchronized } \\
\text { Oscillating } \\
\text { Blowing - } \\
\text { Suction MJ }\end{array}$ & $0.12 / 0.24$ & 30 & $\begin{array}{c}0.6- \\
1.4\end{array}$ & $\begin{array}{c}\text { NACA001 } \\
5\end{array}$ & $2 \times 10^{5}$ & $\begin{array}{c}\text { Experimenta } \\
1 \\
\text { Numerical }\end{array}$ \\
\hline
\end{tabular}

airfoil. The location and geometry of the slot exit in the original design substantially have been preserved; but another jet slot is added next to the original jet slot location to generate synchronized blowing and suction by the translational motion of a piston. Reason for piston type actuator selection is to generate two synthetic jets with 180 degree of phase angle simultaneously. Since the energy consumed for the two synthetic jets obtained in this way is created by a single piston movement, it is approximately the same as the energy consumed for a single jet. Besides, the aim of using dual jets is to increase effectiveness of flow control by increasing jet's area of influence. As a result of this idea, the control effect of actuation on the base flow around the airfoil has been enhanced without making any serious change in the mechanism inside.

Figure 1 shows the solid model of the configuration and a sectional view of the actuation mechanism in the airfoil, respectively. Both rectangular jet slots are placed on the upper surface of the airfoil to allow the jet flows to interact with the separated boundary layer flow easily. A DC motor is used to generate the motion of the piston where the motor output is connected to a crank-connecting rod mechanism hence, the piston is allowed to move back and forth between the dead centers of the compression stroke.

The general working principle of synchronized blowing and suction in one period occurs in two phases. In the first phase, as the motor drives the piston to bottom dead center (to right according to Fig. 1 (b)), the chamber part connected to Slot-2 is pressurized and fluid is accelerated out of the slot exit which is called blowing. At the same time, pressure decreases in the chamber part connected to Slot-1 and fluid moves through the slot into the chamber which is called suction. In the second phase, the piston continues to move to left according to Fig. 
1 (b) and slows down when it approaches the upper dead center of the compression stroke. This time, opposite to the first phase, suction occurs through Slot-2 and fluid is blown through Slot-1. It should be noted that the working principle has an advantage of changing the excitation frequency by adjusting the speed of the DC motor and piston towards the demands in stall control.
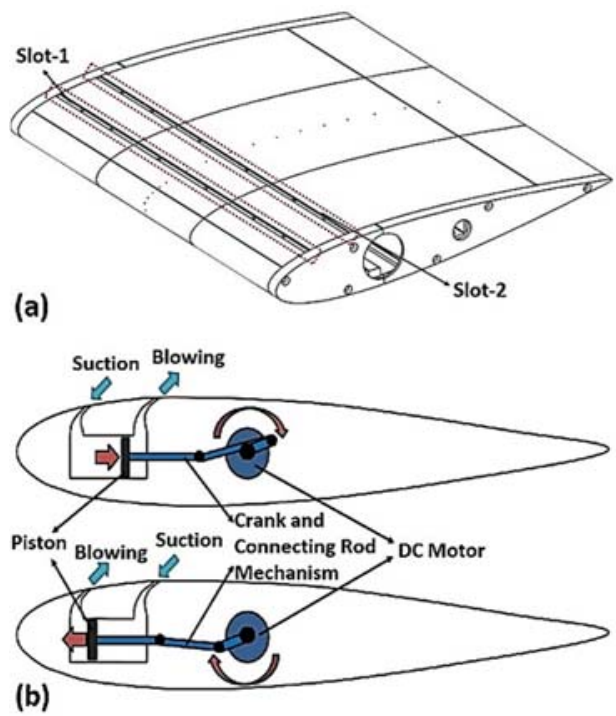

Fig. 1. Jet actuator design: (a) solid model of the configuration, (b) a sectional view of the actuation mechanism in the airfoil.

\section{EXPERIMENTAL STUDIES}

\subsection{Experimental Setup}

There are many design and operational parameters which needed to be clarified before the whole experimental setup could be established. Among the design parameters, location of slots on the airfoil, inclination angles of the slot exits and dimensions of the crank-connecting rod mechanism and piston chamber are crucial in designing the actuation system. On the other hand, jet-to-free stream velocity ratio $\left(R_{j e t}\right)$, AoA and wind tunnel's free stream velocity had to be considered as operational parameters that strongly affect the flow control. In the present study, jet-to-free stream velocity ratio is changed keeping free stream velocity constant at 10 $\mathrm{m} / \mathrm{s}$. Both experimental and numerical studies have been carried out over a wide range AoA to reveal the effect of excitation frequency on stall control.

In the present study, type of the airfoil and Slot-1 with its location and exit inclination angle remained the same as reported by Gilarranz et al. $(2005 \mathrm{a}, \mathrm{b})$. As Fig. 2 indicates, Slot- 1 and Slot- 2 are located respectively in a distance of $12 \%$ and $24 \%$ of the chord length from the leading edge regarding mechanical constraints and mutual assistance to each other as much as possible.

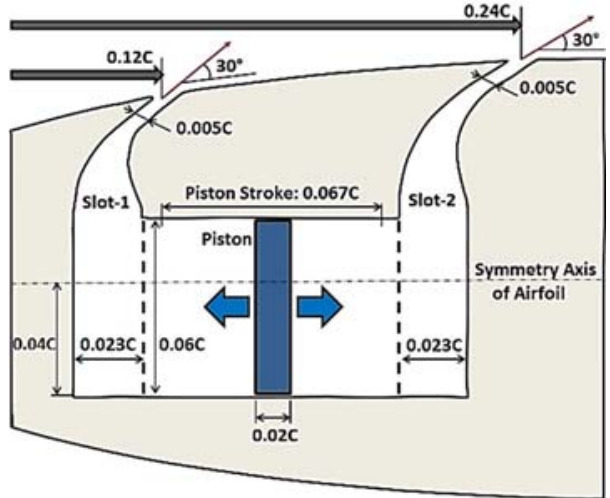

Fig. 2. Main dimensions of jet actuator design.

NACA0015 airfoil has a chord length of $0.30 \mathrm{~m}$, a width of $0.34 \mathrm{~m}$ and for both slot exits the inclination angle to the horizontal was $30^{\circ}$. The subsonic wind tunnel is operated at a Reynolds number of approximately $2 \times 10^{5}$ based on the chord length of the airfoil at standard atmospheric conditions where the density and dynamic viscosity of air can be assumed to be $1.21 \mathrm{~kg} / \mathrm{m}^{3}$ and $1.83 \times 10^{-5} \mathrm{~kg} / \mathrm{ms}$, respectively.

After determining the actuation system and operating conditions, the actuation parameters are defined as the motor speed, maximum jet velocity, nondimensional jet-to-free stream velocity ratio and momentum coefficient for three excitation frequencies as indicated in Table 2. It should be emphasized that these parameters are selected in a way so that $R_{j e t}$ has a value of around 1 as suggested by Gilarranz et al. (2005b).

Table 2 Jet actuator parameters.

\begin{tabular}{|c|c|c|c|c|}
\hline Motor Speed & $\mathrm{f}$ & Ajet & Rjet & $\mathrm{C}_{\mu}$ \\
\hline$(\mathrm{rpm})$ & $(\mathrm{Hz})$ & $(\mathrm{m} / \mathrm{s})$ & & \\
\hline 480 & 8.0 & 6 & 0.6 & 0.0009 \\
\hline 800 & 13.3 & 10 & 1.0 & 0.0025 \\
\hline 1120 & 18.7 & 14 & 1.4 & 0.0049 \\
\hline
\end{tabular}

Airfoil prototype used in the experimental studies is produced from PLA material by a FDM type 3D printer as shown in Fig. 3 (a). The jet actuator mechanism inside the airfoil and the test section of the subsonic wind tunnel are demonstrated in Fig. 3 (a) and Fig. 3 (b), respectively. The prototype consists of the internal jet actuator mechanism and actual airfoil body. A $4 \mathrm{~mm}$ thick steel sheet is used to fix all the PLA components of the airfoil prototype along with a mechanism which can adjust angles of attack. Also, airfoil prototype includes equipment such as PL-3213 model DC motor with encoder, bearings, shafts and various fasteners. After assembling all parts, gaps on the airfoil prototype were closed with clay and entire airfoil surface is covered with adhesive paper for sustaining a smooth surface. 
(a)

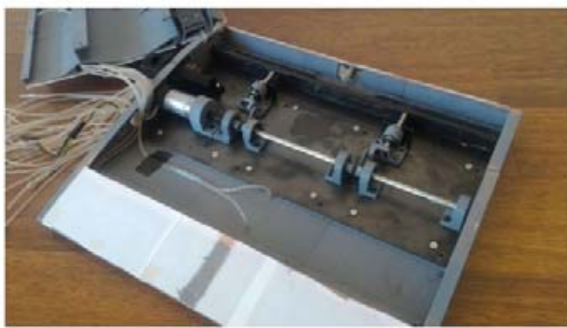

(b)

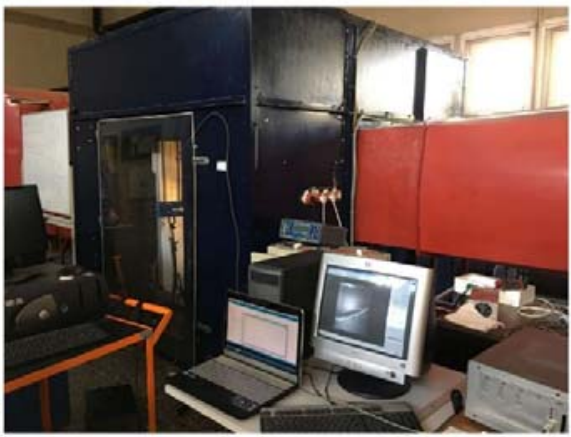

Fig. 3. Experimental setup: (a) jet actuator mechanism inside the airfoil, (b) test section of the subsonic wind tunnel.

In the measurements, pressure taps with an inner diameter of $0.7 \mathrm{~mm}$ are placed at 30 different locations on the mid-section of the airfoil surface to collect pressure data around the airfoil. Pressure taps are connected to a 32-channel ESP-32HD miniature pressure scanner by silicone hoses. It should be mentioned that the pressure taps have been densely placed at locations where pressure changes are expected to be higher.

\subsection{Jet Velocity Measurements}

The wind tunnel experiments have been carried out in Trisonic Research Laboratory of Aeronautics and Astronautics Faculty, Istanbul Technical University. The wind tunnel operates in the incompressible flow range and has open Eiffel type test section. First, the jet velocities are measured at the exits of both slots in a quiescent medium by using DANTEC 90C10 type hot wire anemometer with single-wire probes enabling temporal high precision measurements. Time-dependent velocity data is acquired with a frequency of $15000 \mathrm{~Hz}$ from various points across the slot exits and then reduced to a single timedependent equation by applying ensemble average. Since the jet velocity profiles at the slot exits are identical and close to each other, one representative time-dependent velocity function is found to be sufficient for both slots.

Figure 4 shows the variation of the averaged jet velocities in temporal domain for three excitation frequencies which differ from a single sine or cosine curve. Therefore, a complex function in Eq. (1) is fit to all measured jet velocities. As can be seen from the graphs in Fig. 4, the function of $U_{y}$ is well correlated with experimental data.

$$
\begin{array}{r}
U_{y}(f, t)=\frac{U_{\infty} f}{100}(4.44 \sin (2 \pi f t)-0.48 \cos (2 \pi f t) \\
\left.-2.04 \cos ^{2}(2 \pi f t)+2.04\right)
\end{array}
$$

Figure 5 shows the centered position of the airfoil in the test section of the wind tunnel. To reduce side effects of the tunnel and the blockage factor and provide tightness as much as possible, the spanwise width of the airfoil is chosen as the width of the test section. To be consistent with 2D simulations, edge effects should be avoided thus, the airfoil prototype has been attached on acrylic plates on both ends.

Wind tunnel experiments are conducted between $9^{\circ}$ to $20^{\circ}$ angle of attack and surface pressures have been
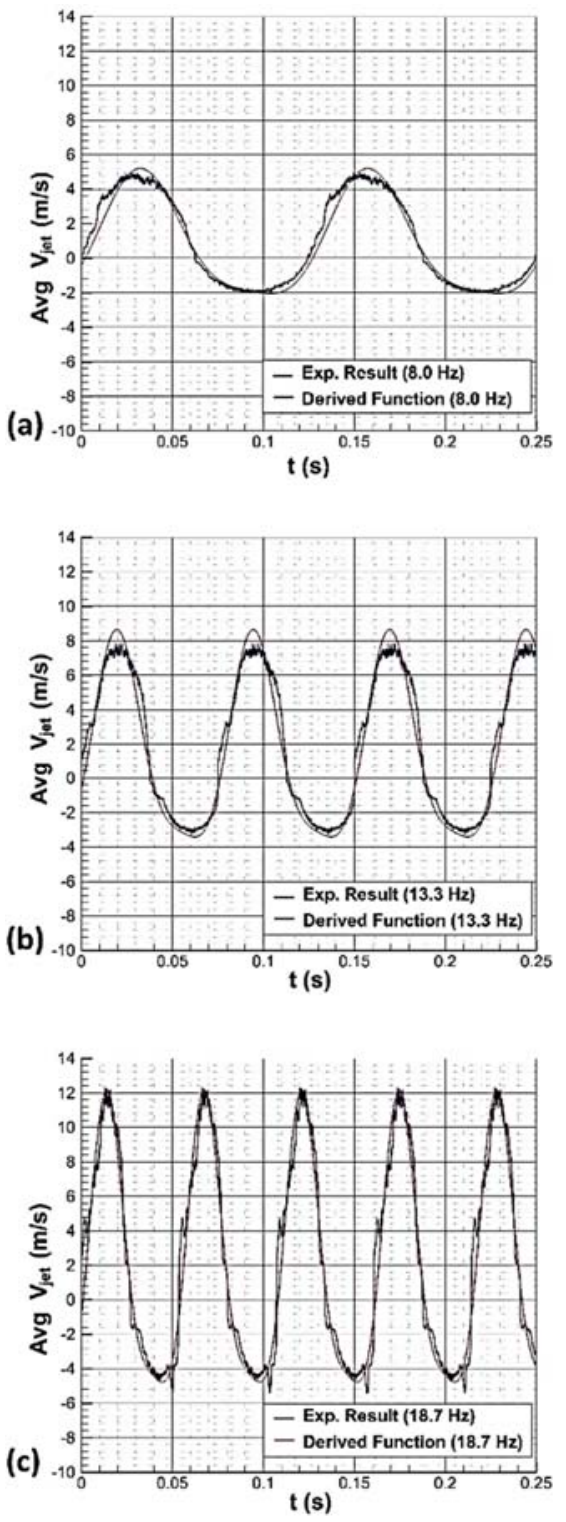

Fig. 4. The averaged jet velocities at slot exits: (a) $f=8.0 \mathrm{~Hz}$, (b) $f=13.3 \mathrm{~Hz}$ and (c) $f=18.7 \mathrm{~Hz}$. 


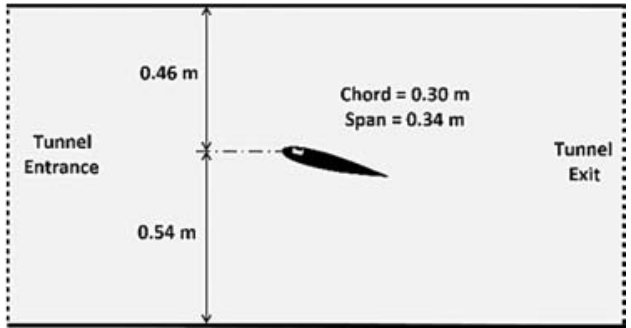

Fig. 5. Position of the airfoil in the test section of the wind tunnel.

measured for the baseline and controlled modes at three excitation frequencies. In all pressure measurements data was collected with a frequency of $128 \mathrm{~Hz}$ and averaged over 20 seconds. To ensure repeatability and accuracy of the measurements, the experiments are repeated at least twice.

\section{COMPUTATIONAL APPROACH}

\subsection{Governing Equations}

In the CFD simulations the conservation equations for mass and momentum for 2D, transient and fully turbulent flow are solved together with the turbulence transport equations by a finite volumebased commercial code ANSYS-Fluent where pressure based flow solver is selected since the flow is incompressible.

The continuity, Navier-Stokes equations and the stress tensor are given in Eq. (2), Eq. (3) and Eq. (4), respectively.

$$
\begin{aligned}
& \frac{\partial \rho}{\partial \mathrm{t}}+\nabla \cdot(\rho \vec{v})=0 \\
& \frac{\partial}{\partial \mathrm{t}}(\rho \vec{v})+\nabla \cdot(\rho \vec{v} \vec{v})=-\nabla p+\nabla \cdot(\overline{\bar{\tau}})+\rho \vec{g} \\
& \overline{\bar{\tau}}=\mu\left[\left(\nabla \vec{v}+\nabla(\vec{v})^{\mathrm{T}}\right)-\frac{2}{3} \nabla \cdot \vec{v} \mathrm{I}\right]
\end{aligned}
$$

In CFD solver, coupled algorithm is selected and the governing equations are solved iteratively until the convergence criteria have been satisfied and the residuals dropped below $10^{-5}$.

The turbulence models are classified generally in three groups: RANS-based models, LES and hybrids of both. Compared to each other, these models have superiorities and disadvantages in terms of computational effort and accuracy and of the solution. The most important issue in LES is high computational cost and in connection with it, the need for high performance computing for 3D transient solutions. This is mostly not so challenging in CFD computations with RANS-based turbulence models. Furthermore, LES and DES models are sensitive to the dimension of the computational domain which is a parameter strictly affecting the robustness of the solution. A major drawback of the

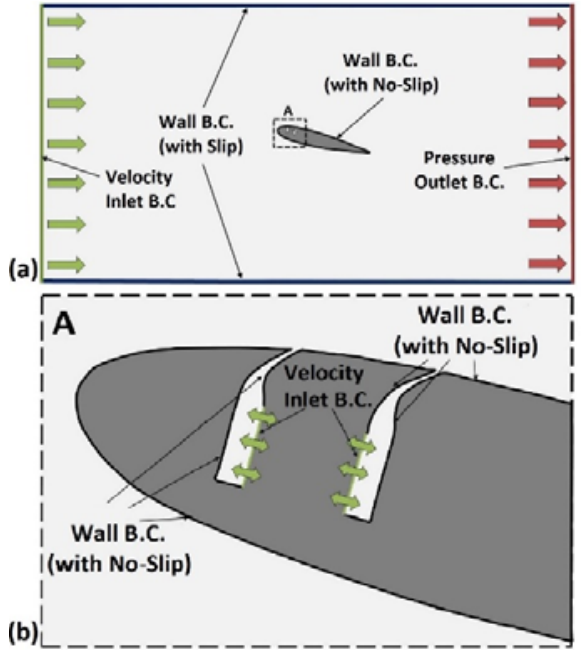

Fig. 6. Boundary conditions for: (a) wind tunnel, (b) jet actuator.

RANS-based turbulence models is that they partially estimate flow separation and cannot succeed in predicting transient vortex shedding as LES and DES models do. 3D analysis is shown to be much more successful than 2D analysis in accurately detecting vortex shedding and separation bubble, depending on mesh resolution in span-wise direction (Schmidt and Thiele 2003).

Schmidt and Thiele (2003) compared the performance of RANS and DES to quantify the influence of transient flow patterns over an airfoil. They reported that 3D simulations could predict unsteady flow features in stall development much better than 2D simulations, adding that 2D DES was leading to results comparable to those obtained by 2D RANS. Being aware of this and considering the limited computational cost within the scope of this study, it was decided to carry out numerical studies with the 2D DES model.

The DES model is a blend of RANS-based turbulence model with LES, where an appropriate RANS model should be selected. In the current study, SST k- $\omega$ turbulence model is preferred among other turbulence models in order to predict flow separation more accurately as proposed by Kim and Kim (2009). The transport equation for the SST k- $\omega$ model are shown in Eq. (5) and Eq. (6) (ANSYS FLUENT 2013).

$$
\begin{gathered}
\frac{\partial}{\partial \mathrm{t}}(\rho \mathrm{k})+\frac{\partial}{\partial \mathrm{x}_{\mathrm{i}}}\left(\rho \mathrm{ku} \mathrm{u}_{\mathrm{i}}\right)= \\
\frac{\partial}{\partial \mathrm{x}_{\mathrm{j}}}\left(\Gamma_{\mathrm{k}} \frac{\partial \mathrm{k}}{\partial \mathrm{x}_{\mathrm{j}}}\right)+\mathrm{G}_{\mathrm{k}}-\mathrm{Y}_{\mathrm{k}}+\mathrm{S}_{\mathrm{k}} \\
\frac{\partial}{\partial \mathrm{t}}(\rho \omega)+\frac{\partial}{\partial \mathrm{x}_{\mathrm{i}}}\left(\rho \omega \mathrm{u}_{\mathrm{j}}\right)= \\
\frac{\partial}{\partial \mathrm{x}_{\mathrm{j}}}\left(\Gamma_{\omega} \frac{\partial \omega}{\partial \mathrm{x}_{\mathrm{j}}}\right)+\mathrm{G}_{\omega}-\mathrm{Y}_{\omega} \\
+\mathrm{S}_{\omega}+\mathrm{D}_{\omega}
\end{gathered}
$$


The details of the SST k- $\omega$ DES model can be found in ANSYS Theory Guide (ANSYS FLUENT 2013).

\subsection{Boundary Conditions}

Figure 6 (a) shows the real boundary conditions during tests that have been applied to all CFD calculations. Airfoil walls are defined by no-slip boundary conditions. Test section of the wind tunnel is actually a limitation. However, the boundaries have been assigned as slip-walls for reducing the analysis cost, because the effect of the lower and upper boundaries on the flow field around the airfoil is negligible. The inlet of the domain is subjected to constant free stream velocity at $10 \mathrm{~m} / \mathrm{s}$ and the outlet is prescribed by pressure outlet boundary condition.

As suggested by You and Moin (2009), to save computational effort, the motion of the piston is defined by time-dependent velocity profile instead of using dynamic mesh approach. The measured velocities across the exits of the slots are converted to functions $U_{1}$ in Eq. (7) and $U_{2}$ in Eq. (8) and then transferred to piston surfaces as shown in Fig. 6 (b). Phase shift between these functions defined by half a period (1/2f) enabled piston's motion in opposite direction during operation. Hence, these functions in the form of UDF boundary conditions can mimic suction at one piston's surface and blowing at the other surface of the piston, simultaneously.

(a)
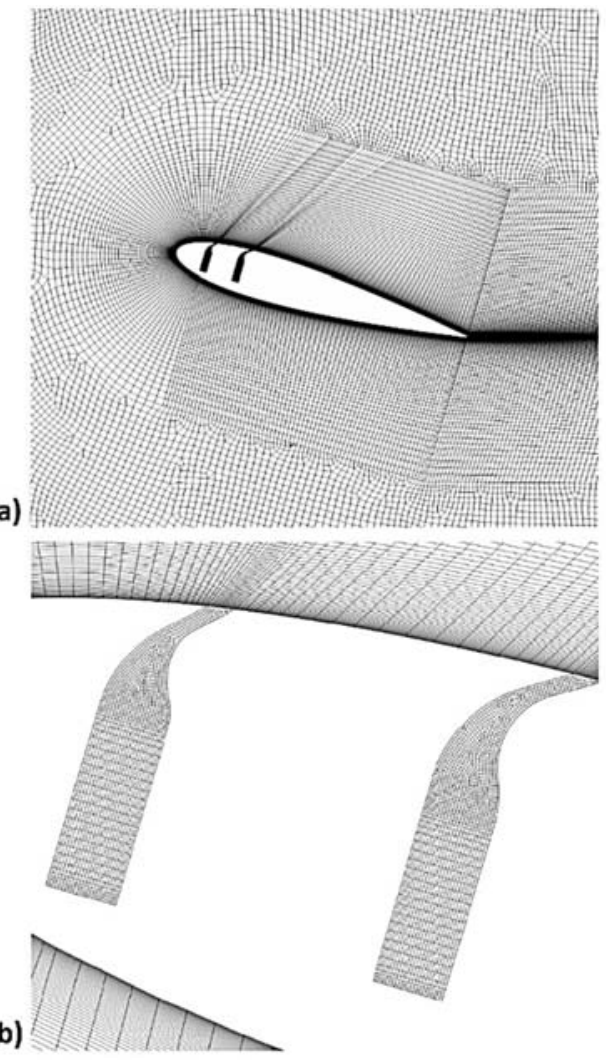

Fig. 7. Mesh for: (a) around the airfoil and (b) around the jet actuator.

$$
\begin{array}{r}
U_{1}(f, t)=\frac{U_{\infty} f}{100}(3.7 \sin (2 \pi f t)-0.4 \cos (2 \pi f t) \\
\left.-1.7 \cos ^{2}(2 \pi f t)+1.7\right) \\
U_{2}(f, t)=\frac{U_{\infty} f}{100}\left(3.7 \sin \left(2 \pi f\left(t-\frac{1}{2 f}\right)\right)\right. \\
-0.4 \cos \left(2 \pi f\left(t-\frac{1}{2 f}\right)\right)-1.7 \cos ^{2}\left(2 \pi f\left(t-\frac{1}{2 f}\right)\right)
\end{array}
$$

\subsection{Mesh Generation and Mesh Independency Tests}

Meshed domains around the airfoil and actuator slots are shown in Fig. 7. In the boundary layer and flow separation regions and also in the vicinity of the slots a refined mesh is used so that $y^{+}$was kept at around 1 in accordance with requirements of the turbulence model. Parts of the computational domain far from the airfoil have been meshed less dense with the aim not to increase computational effort in the freestream regions.

In the transient analysis, the time step size was chosen $10^{-4}$ seconds which is compatible with the time step size reported in You and Moin (2009). The computations have been performed with that time step and they continued approximately 1 second.

(a)
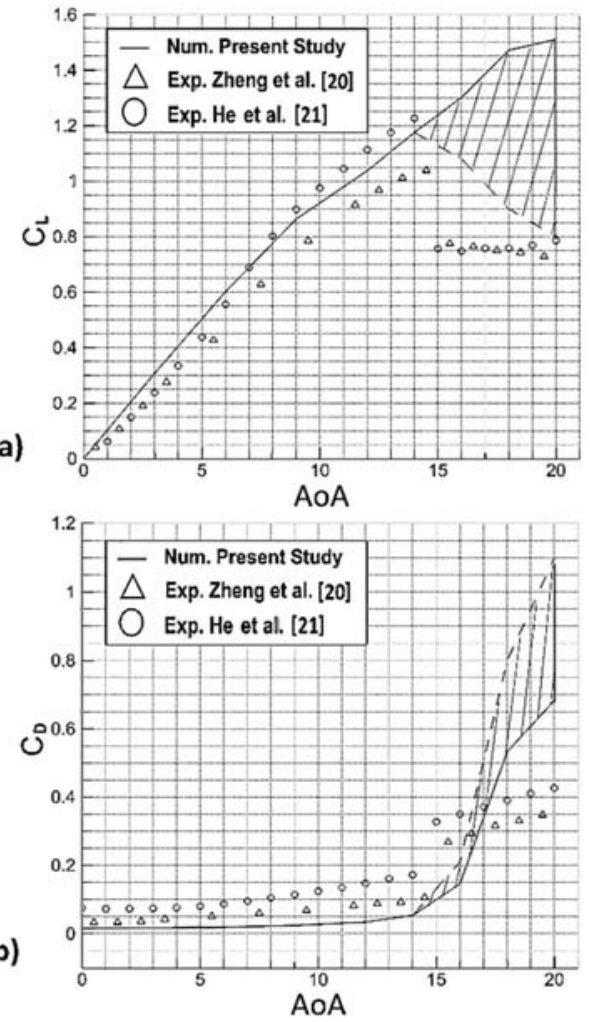

Fig. 8. Validation of averaged force coefficients from CFD analysis with literature results for the NACA0015 airfoil: (a) $C_{L}$ vs AoA and (b) $C_{D}$ vs AoA. (Hatched regions show fluctuations of force coefficients because of stall.) 
Table 3 Lift and drag coefficients in the mesh independency tests for the baseline airfoil.

\begin{tabular}{|c|c|c|c|c|c|c|c|c|}
\hline \multirow{2}{*}{$\begin{array}{c}\text { AoA } \\
\left({ }^{\circ}\right)\end{array}$} & \multicolumn{2}{|c|}{ Grid-1 } & \multicolumn{2}{c|}{ Grid-2 } & \multicolumn{2}{c|}{ Grid-3 } & \multicolumn{2}{c|}{ Grid-4 } \\
\cline { 2 - 9 } & $\mathrm{C}_{\mathrm{L}}$ & $\mathrm{C}_{\mathrm{D}}$ & $\mathrm{C}_{\mathrm{L}}$ & $\mathrm{C}_{\mathrm{D}}$ & $\mathrm{C}_{\mathrm{L}}$ & $\mathrm{C}_{\mathrm{D}}$ & $\mathrm{C}_{\mathrm{L}}$ & $\mathrm{C}_{\mathrm{D}}$ \\
\hline 0 & 0.00095 & 0.01591 & 0.00019 & 0.01593 & 0.00055 & 0.01595 & 0.00062 & 0.01593 \\
\hline 3 & 0.30806 & 0.01659 & 0.30566 & 0.01662 & 0.28575 & 0.01658 & 0.28252 & 0.01659 \\
\hline 6 & 0.60176 & 0.01894 & 0.59677 & 0.01883 & 0.56941 & 0.01873 & 0.56328 & 0.01880 \\
\hline 9 & 0.86382 & 0.02438 & 0.85288 & 0.02418 & 0.83162 & 0.02377 & 0.82111 & 0.02386 \\
\hline 12 & 1.03749 & 0.03415 & 1.03591 & 0.03390 & 1.05132 & 0.03461 & 1.06122 & 0.03554 \\
\hline 14 & 1.17537 & 0.05381 & 1.14618 & 0.05113 & 1.11425 & 0.04927 & 1.06794 & 0.04725 \\
\hline \multicolumn{7}{|c|}{ Number of elements: Grid-1 (100000), Grid-2 (75000), Grid-3 (50000) and Grid-4 (25000). } \\
\hline
\end{tabular}

Mesh independency tests have been carried out for the baseline airfoil between $0^{\circ}$ to $14^{\circ}$ angles of attack. In all the different dense meshes, surface $y^{+}$ distributions are kept constant in the desirable ranges. Table 3 summarizes the convergence of the lift and drag coefficients in mesh independency analysis. As a result of the mesh tests, a mesh with around 100.000 number of elements for baseline airfoil is found sufficient. On the other hand, the number of necessary elements for the controlled airfoil with jet actuator is approximately 150.000 because of the chamber and slots inside the airfoil.

\section{RESULTS}

\subsection{Lift and Drag Coefficients Evaluation}

For the baseline NACA0015 airfoil, the change of lift $\left(C_{L}\right)$ and drag $\left(C_{D}\right)$ coefficients in the whole range of AoA between $0^{\circ}$ to $20^{\circ}$ have been obtained. Figure 8 shows a comparison of the current numerical results with the experimental data in literature. Averaged CFD results in the present study have been validated against lift $\left(C_{L}\right)$ and drag coefficients $\left(C_{D}\right)$ obtained between $0^{\circ}$ to $20^{\circ}$ AoA from two experimental studies conducted by Zheng et al. (2018) and He et al. (2009). It is found that the CFD results agree well with the experimental data to a large extent, however after $15^{\circ}$ AoA stall affects both lift and drag coefficients and the difference has become evident.

Figure 9 shows the variation of averaged $C_{L}$ and $C_{D}$ coefficients at three excitation frequencies for $16^{\circ}$ and $18^{\circ}$ angles of attack. As these CFD results reveal, with increasing excitation frequency, active flow control provides lift enhancement by controlling stall hence, $C_{L}$ has increased as well. Contrary to $C_{L}$, with increasing excitation frequency, $C_{D}$ decreases. Active flow control is shown to manipulate flow separation zone by transporting momentum to the boundary layer by simultaneously generated jets and this can avoid the negative effects of stall on lift forces. This active flow control is numerically shown as an effective method capable of enhancing lift and reducing drag at the same time.

\subsection{Surface Pressure Evaluation}

In both experimental and numerical studies, pressure coefficient $\left(C_{P}\right)$ distributions on the pressure and suction surfaces of the airfoil are evaluated as the main indicator for flow separation control, its delay or prevention. CFD analysis give the opportunity to comment on the changes in averaged lift and drag coefficients with respect to AoA in both baseline flow and controlled modes.

Figure 10 demonstrates a wide perspective about the effect of jet actuator on flow separation control in the whole frequency and AoA spectrum. The wind tunnel experiments have shown that flow control cannot provide significant influence on $\mathrm{CP}$ distribution around the airfoil at $9 \circ \mathrm{AoA}$ and is
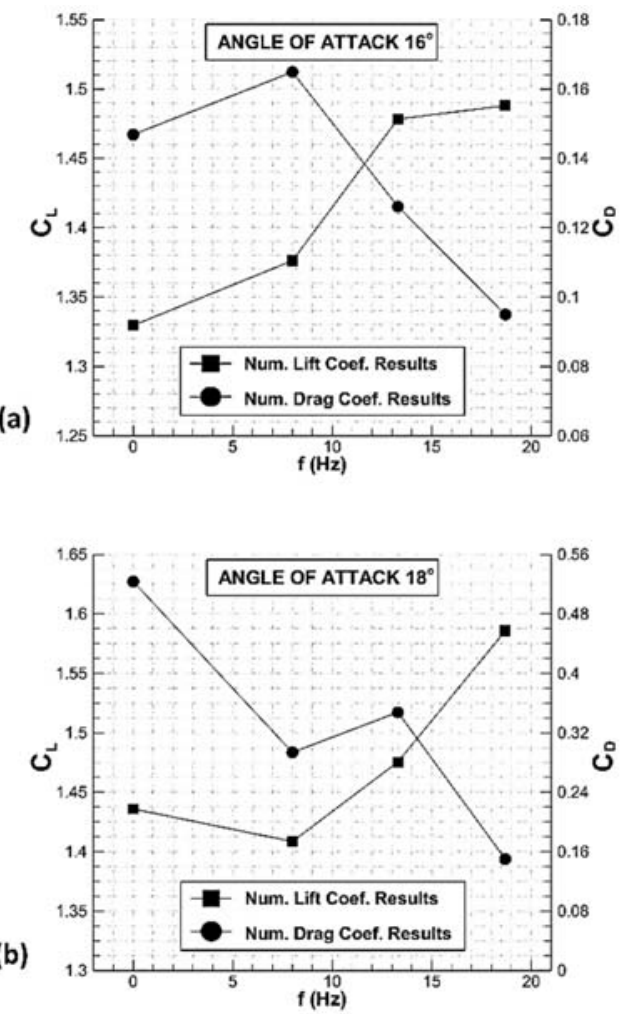

Fig. 9. Variation of averaged $C_{L}$ and $C_{D}$ coefficients at three excitation frequencies for $16^{\circ}$ and $18^{\circ}$ angles of attack. 

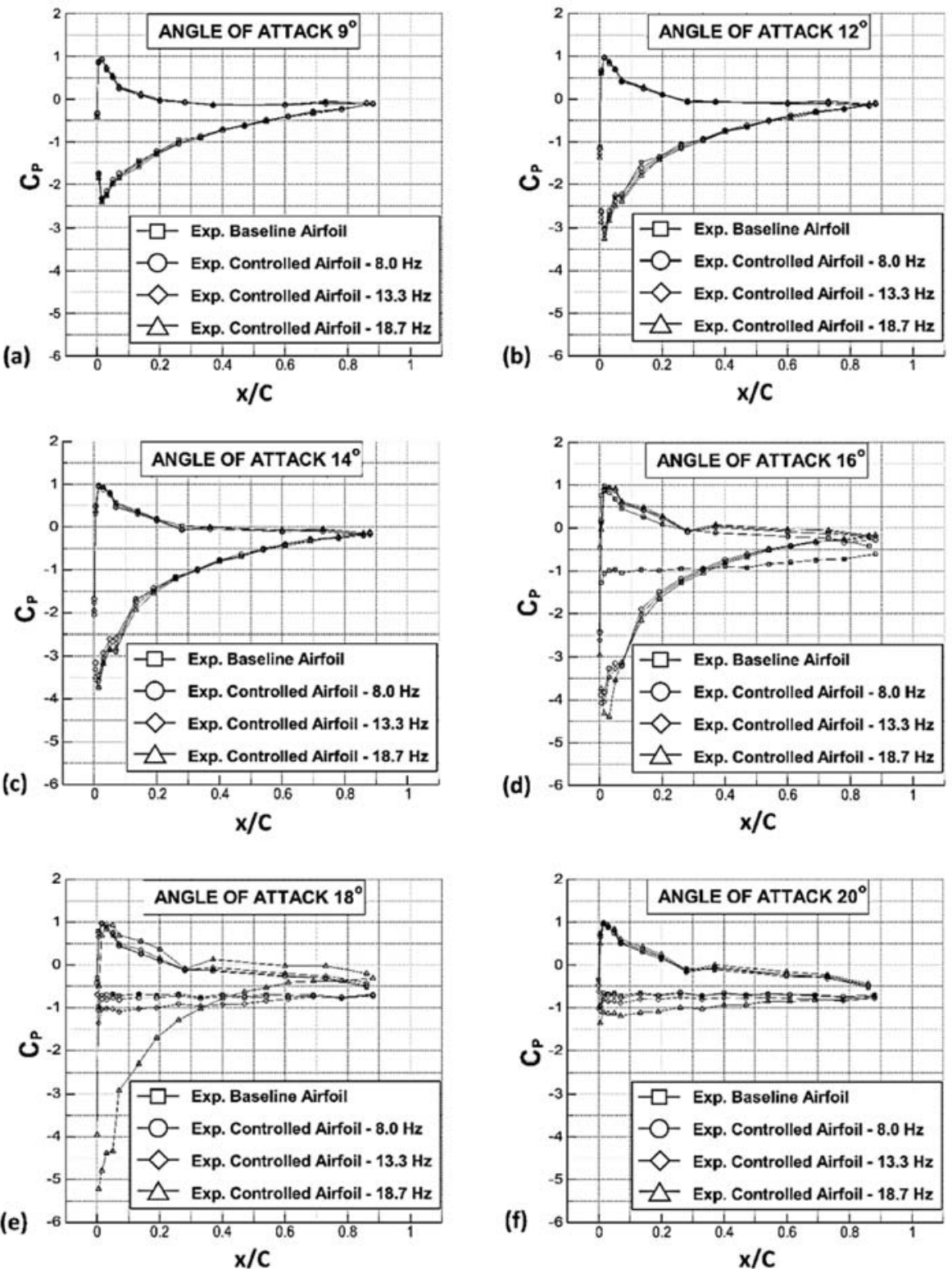

Fig. 10. Surface pressure measurements at various AoAs in experimental study: (a) $9^{\circ}$, (b) $12^{\circ}$, (c) $14^{\circ}$, (d) $16^{\circ}$, (e) $18^{\circ}$, (f) $20^{\circ}$.

independent of the excitation frequency (see Fig. 10 (a)). For $12^{\circ}$ and $14^{\circ}$ AoA, the influence of the flow control has become slightly appreciable especially near the leading edge of the airfoil (see Fig. 10 (b) and $10(\mathrm{c})$ ). But the remarkable effect of active flow control has been detected at $16^{\circ} \mathrm{AoA}$ where stall can be delayed at all excitation frequencies as indicated in Fig. 10 (d). At this angle of attack, $C_{P}$ distribution around the airfoil has increased considerably especially near the leading edge up to $\mathrm{x} / \mathrm{c}=0.4$ since the jet slot exits' locations are at $\mathrm{x} / \mathrm{c}=0.12$ and $\mathrm{x} / \mathrm{c}=$ 0.24 .

It is observed that the effectiveness in delaying stall depends on AoA and the excitation frequency as well. This fact is obvious at $18^{\circ} \mathrm{AoA}$, since excitation frequencies of $8.0 \mathrm{~Hz}$ and $13.3 \mathrm{~Hz}$ have been found insufficient to delay stall. However, if the jet actuator is operated at $18.7 \mathrm{~Hz}$, it succeeds in stall control and provides considerably high $C_{P}$ values from the leading edge up to $\mathrm{x} / \mathrm{c}=0.4$ as indicated in Fig. 10 (e).

As Fig. 10 (f) shows, the jet actuator cannot succeed in stall control at any excitation frequency for $20^{\circ}$ AoA. At such high AoA, the system is not capable of inserting sufficient jet-momentum into the separated boundary layer thus, stall cannot be taken under control at all. Among all operating conditions, based on the performance of the jet actuator, $16^{\circ}$ and $18^{\circ}$ AoA are considered worth to be compared with CFD simulations. 
(a)
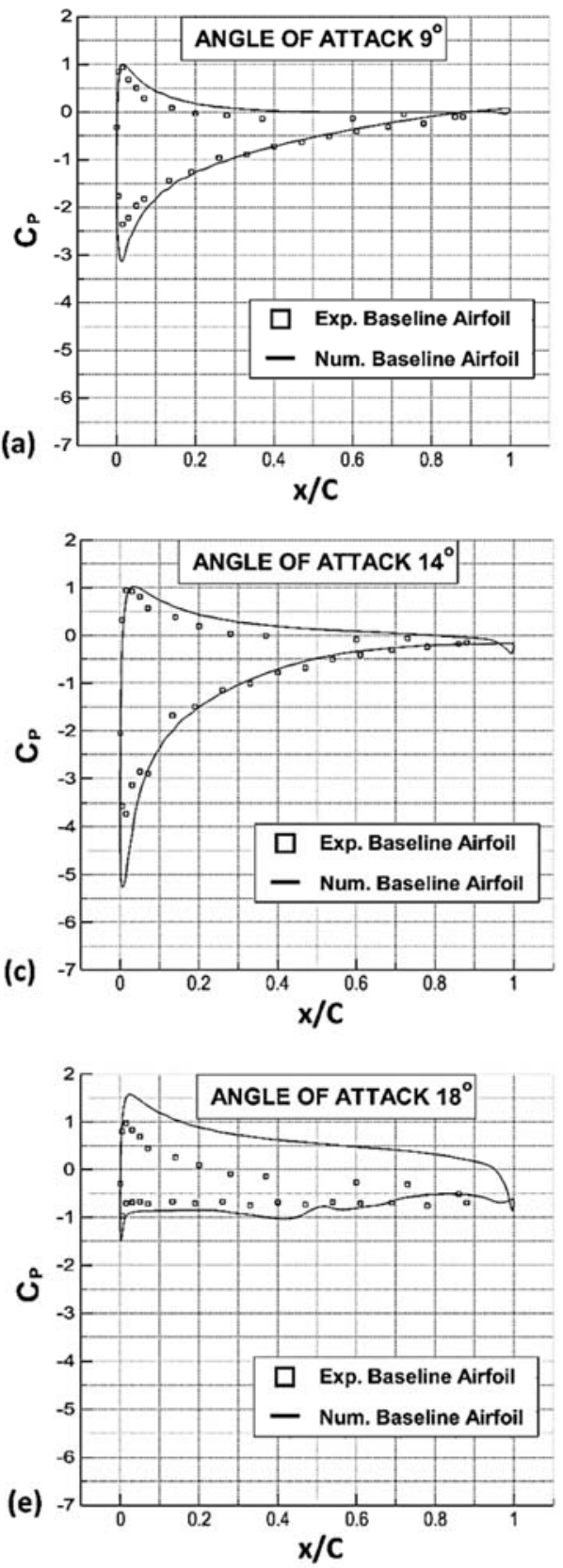
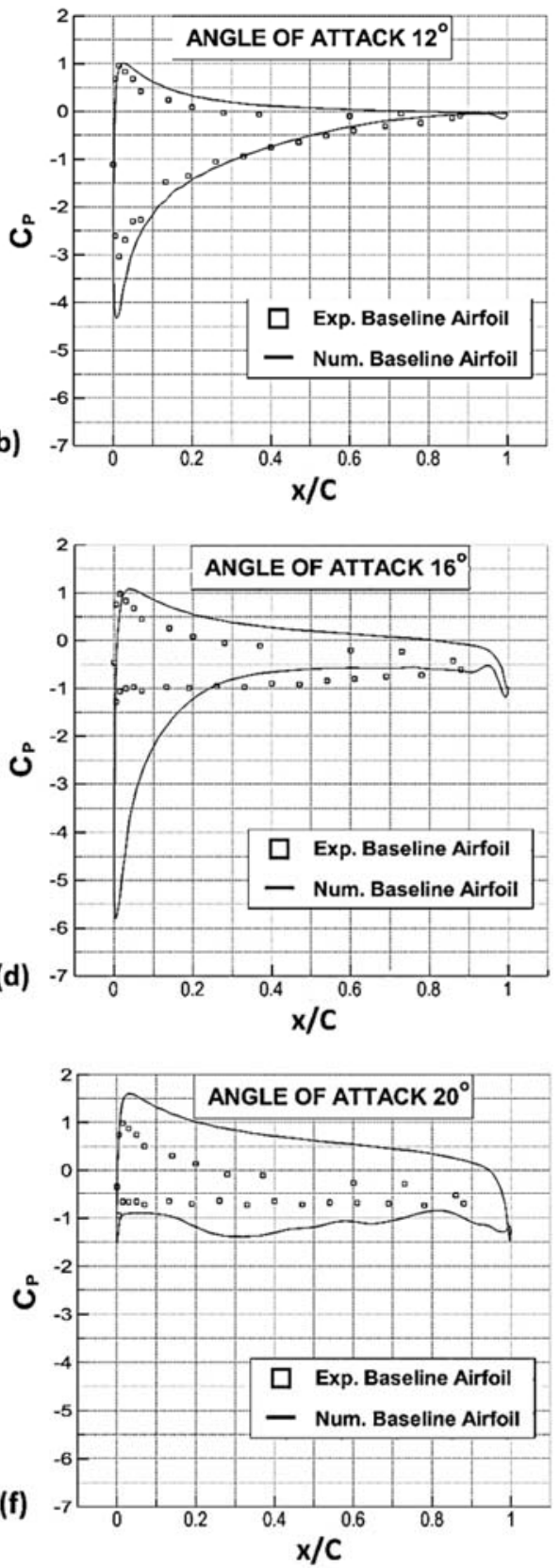

Fig. 11. Comparison of surface pressure measurements with CFD results at various AoAs in uncontrolled mode: (a) $9^{\circ}$, (b) $12^{\circ}$, (c) $14^{\circ}$, (d) $16^{\circ}$, (e) $18^{\circ}$ and (f) $20^{\circ}$.

Figure 11 shows the comparison of numerically and experimentally obtained $C_{P}$ distributions over the whole range of AoA between $9^{\circ}$ to $20^{\circ}$ for baseline airfoil. In the uncontrolled mode, if the operating conditions are at low AoA such as between $9^{\circ}$ to $14^{\circ}$, a compatible match is found between CFD results and experiments (see Fig. 11 (a) to Fig. 11 (c)). However, with increasing AoA, stall initiates at $16^{\circ}$ AoA and the numerical model cannot succeed in approaching the experimental data especially near the leading edge (see Fig. 11 (d)). Further increasing the AoA to $18^{\circ}$ and $20^{\circ}$, the CFD model over-predicts local $C_{P}$ with respect to measurements especially on pressure side of the airfoil (see Fig. 11 (e) to Fig. 11 (f)). The deficiencies should be attributed to the usage of a $2 \mathrm{D}$ domain where vortex shedding and other 3D phenomena related to flow separation cannot be successfully captured. Despite this, in some manner the trend of the overall $C_{P}$ distributions at such high AoA is satisfactorily adequate. 

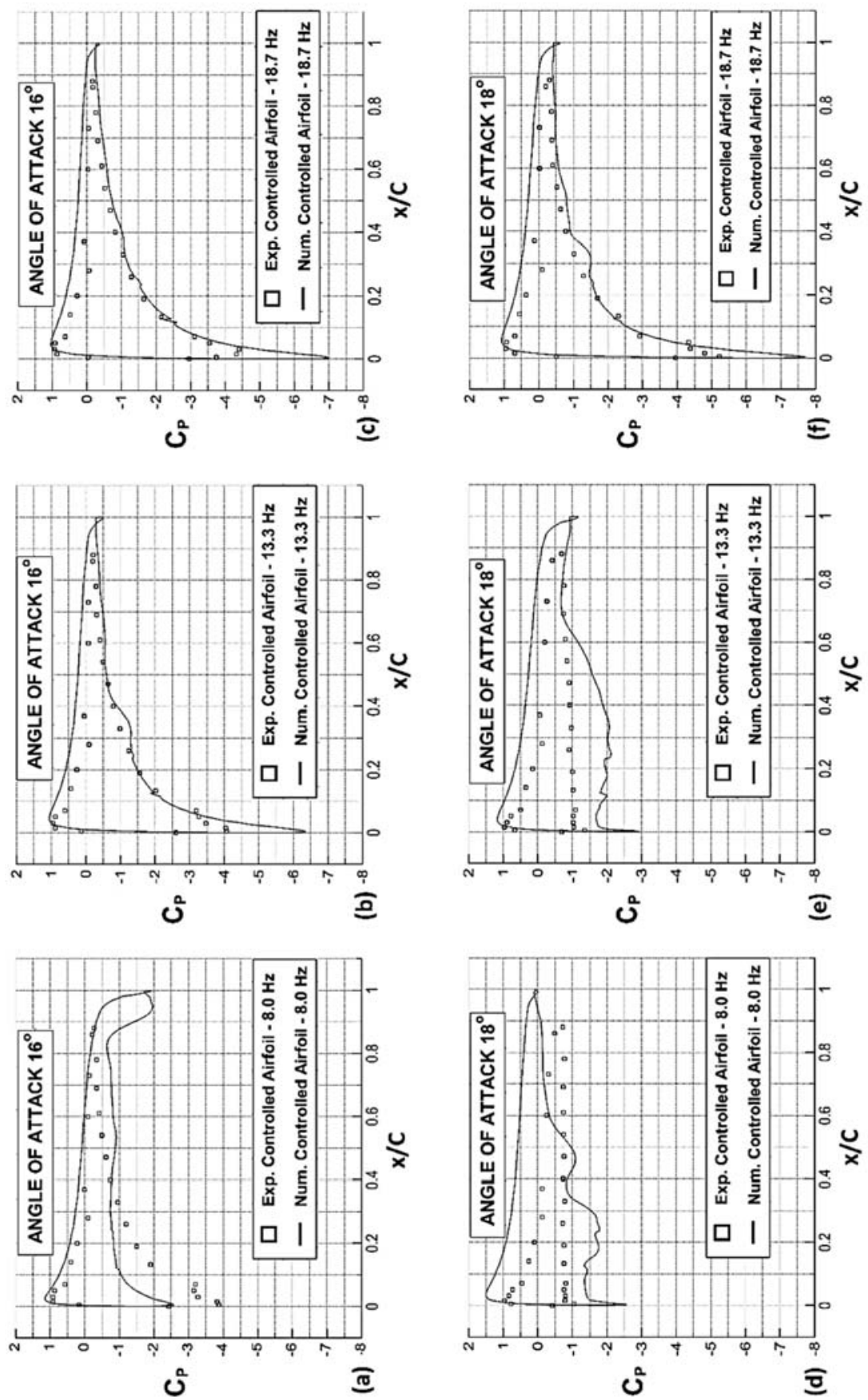

Fig. 12. Comparison of surface pressure measurements with CFD results at various AoAs in controlled modes: (a) $\mathrm{AoA}=16^{\circ}$ and $\mathrm{f}=8.0 \mathrm{~Hz}$, (b) $\mathrm{AoA}=16^{\circ}$ and $\mathrm{f}=13.3 \mathrm{~Hz}$, (c) $\mathrm{AoA}=16^{\circ} \mathrm{f}=18.7 \mathrm{~Hz}$, (d) $\mathrm{AoA}=$ $18^{\circ} \mathrm{f}=8.0 \mathrm{~Hz}$, (e) $\mathrm{AoA}=18^{\circ} \mathrm{f}=13.3 \mathrm{~Hz}$, (f) $\mathrm{AoA}=18^{\circ} \mathrm{f}=18.7 \mathrm{~Hz}$.

Figure 12 (a) to Fig. 12 (f) demonstrate the effect of jet actuator on stall control and comparison between experimental and CFD results for controlled mode at $16^{\circ}$ and $18^{\circ}$ AoA. Among all excitation frequencies, $8.0 \mathrm{~Hz}$ is found to be least effective one to control stall, in addition to that, the CFD results at this frequency cannot be validated against experiments satisfactorily, mainly at the trailing edge of the airfoil (see Fig. 12 (a)). As Fig. 12 (b) and Fig. 12 (c) indicate, both CFD simulations and wind tunnel experiments agree well with each other and the jet actuator achieves stall control at the excitation frequencies of 13.3 and $18.7 \mathrm{~Hz}$. This should be associated to the corresponding jet-to free stream velocity ratios of 1 and 1.4, since stall is more likely to be influenced by relatively high jet velocities as the wind tunnel experiments have revealed. 

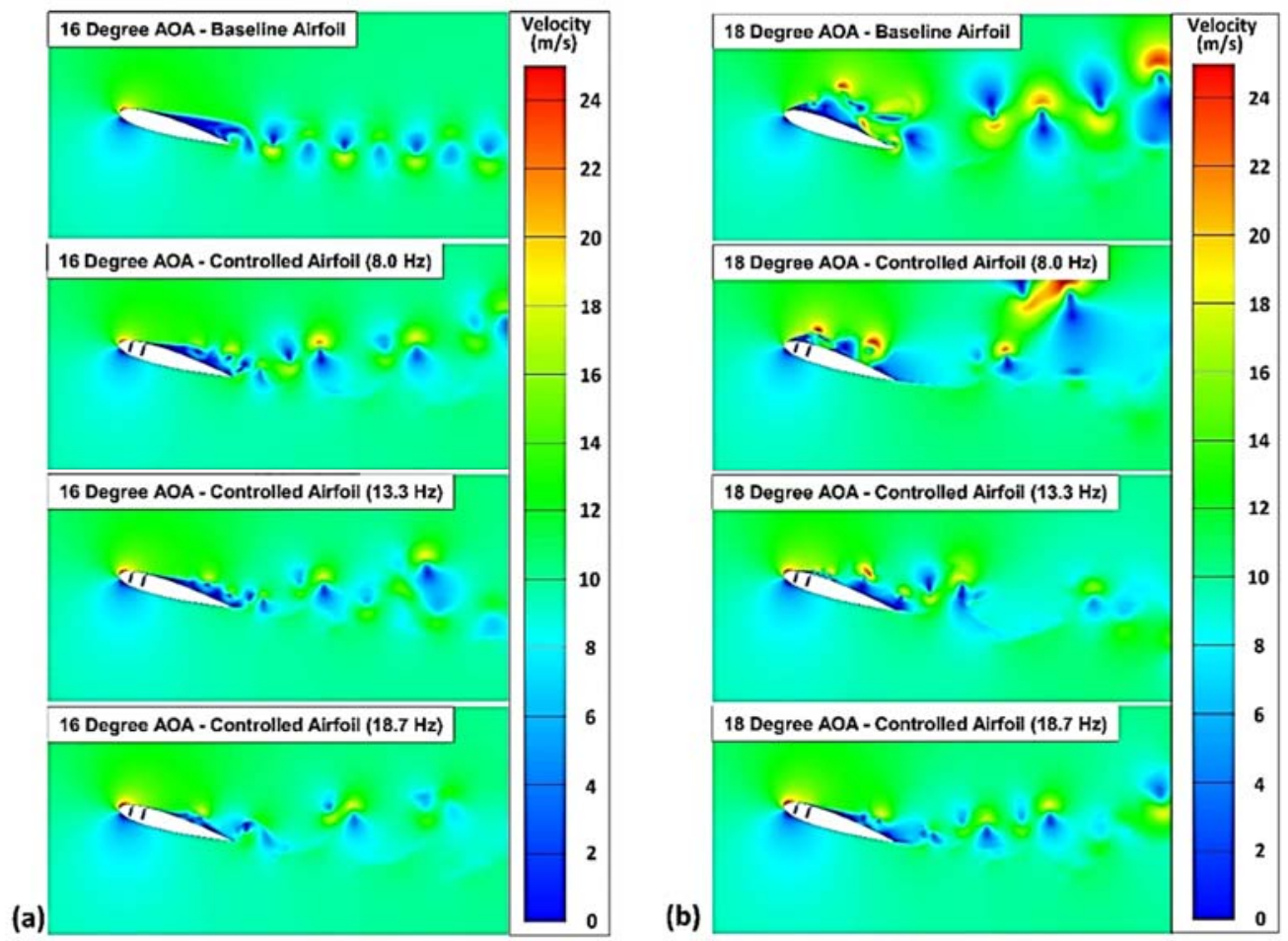

Fig. 13. Instantaneous velocity magnitude contours for different control frequencies at: (a) $\mathrm{AoA}=16^{\circ}$, (b) $\mathrm{AoA}=18^{\circ}$.

It should be underlined that angle of attack is playing the most significant role in stall control and this is proven by $18^{\circ}$ AoA as Fig. 12 (d) and Fig. 12 (e) show. At such an angle of attack, stall cannot be avoided if the jet actuator is adjusted to the $8.0 \mathrm{~Hz}$ and $13.3 \mathrm{~Hz}$ excitation frequencies. As proven numerically and experimentally in Fig. 12 (f), active flow control achieves stall delay only if dominant governing parameters such as jet-to-free stream velocity ratio and AoA have been chosen compatible with each other.

\subsection{Velocity Magnitude Evaluation}

Figure 13 shows the velocity contours of the selected cases with stall control mentioned in previous sections. Figure 13 (a) and Fig. 13 (b) represent instantaneous velocity magnitudes for the baseline flow and controlled cases and provide a deep insight into the flow structures which are not observable during experiments. As Fig. 13 (a) indicates, at $16^{\circ}$ AoA, baseline airfoil flow is separated exactly at the location of Slot-2 $(\mathrm{x} / \mathrm{c}=0.24)$ and if the jet actuator is operated at a certain excitation frequency, flow separation zone on the upper surface of the airfoil becomes smaller. With increasing excitation frequency and corresponding jet-momentum transport into the separated boundary layer, the velocity profile just downstream of Slot-2 is reconstructed indicating that flow separation has been delayed.

It should be mentioned that an unseparated velocity profile is achieved in the vicinity of $x / c=0.3$ if flow separation at $16^{\circ} \mathrm{AoA}$ is subjected to an excitation frequency of $13.3 \mathrm{~Hz}$. When the excitation frequency is set to $18.7 \mathrm{~Hz}$, flow reattachment occurs at approximately $\mathrm{x} / \mathrm{c}=0.4$ under the influence of jetmomentum transport hence, the boundary layer velocity profile can be reestablished.

Figure 13 (b) shows that at $18^{\circ} \mathrm{AoA}$, flow has started to separate almost at the stagnation point of the airfoil. As a result, a considerably big separation zone occurs on the whole upper surface which makes flow control under any circumstance complicated. Excitation frequencies of $8.0 \mathrm{~Hz}$ and $13.3 \mathrm{~Hz}$ can partially be effective in reducing the big separation zone. However, increasing the excitation frequency of $18.7 \mathrm{~Hz}$ has been found to be effective in delaying flow separation and controlling stall. If the jet actuator is operated at $18.7 \mathrm{~Hz}$, flow reattachment can be obtained at approximately $\mathrm{x} / \mathrm{c}=0.35$.

It should be mentioned that distance between jetslots is a parameter that affects flow control. Because of the restraints of actuator design, this parameter should be constant. Also, specific to present design, jet exit frequency is directly related to piston motion. Since piston stroke is constant, high frequency corresponds to high momentum coefficient in this system and they are not independent variables.

\section{CONCLUSION}

In this study, a novel fluidic jet actuator was investigated experimentally and numerically to delay flow separation and control stall on a NACA0015 airfoil at high angles of attack. The jet actuator was 
driven by a piston mechanism and operates at $8.0 \mathrm{~Hz}$, 13.3 $\mathrm{Hz}$ and $18.7 \mathrm{~Hz}$ where a synchronized jet flow was generated by blowing and suction at the dual exits of the actuator slots. The experiments have been conducted in a subsonic wind tunnel at a constant free stream velocity and the CFD simulations were performed with ANSYS-Fluent using the blend of 2D unsteady SST k- $\omega$ and DES turbulence model.

The effect of jet actuator was tested and evaluated experimentally and numerically in terms of surface pressure distributions. It is worth to mention that the results were interpreted by frequency rather than by the wavelength of disturbances which can be regulated by using second slot position. The model and actuator design did not give possibility to easily modify slot position in order to examine the variations in flow behavior with slot distance. It was seen that the uncontrolled baseline flow enters stall at $16^{\circ} \mathrm{AoA}$ and could be recovered at all excitation frequencies. Besides, the effect of active flow control by synchronized blowing and suction was examined numerically at $16^{\circ}$ and $18^{\circ}$ AoA and generally an increase in $C_{L}$ and decrease in $C_{D}$ have been observed. This suggested that this active flow control method with synchronized blowing and suction was useful in lift enhancement and drag reduction.

In numerical studies, the verification of the results by comparing them with the results in the literature is an important basis. When the airfoil in uncontrolled mode was subjected to flow between $9^{\circ}$ to $14^{\circ} \mathrm{AoA}$, a convincing match was found between numerically and experimentally obtained $C_{P}$. On the other hand, if the airfoil in controlled mode was subjected to flow at $16^{\circ}$ and $18^{\circ} \mathrm{AoA}$, a satisfactory match was found between numerically and experimentally obtained $C_{P}$ in most cases. The deviations in some cases have been thought to be caused by the simplified 2D model.

In this study, the effect of jet actuator on stall control was revealed for different AoAs and frequencies. At $18^{\circ} \mathrm{AoA}$, the controlled mode only at $18.7 \mathrm{~Hz}$ achieved stall control, but at $20^{\circ}$ AoA, active flow control was found insufficient at all frequencies at all. This indicated that stall control with synchronized blowing and suction was effective in the range of $14^{\circ} \leq \mathrm{AoA} \leq 20^{\circ}$.

\section{REFERENCES}

ANSYS FLUENT (2013). ANSYS Fluent Theory Guide.

Buchmann, N. A., C. Atkinson and J. Soria (2013). Influence of ZNMF jet flow control on the spatio-temporal flow structure over a NACA0015 airfoil. Experiments in Fluids 54(3).

Cattafesta, L. N. and M. Sheplak (2011). Actuators for active flow control. Annual Review of Fluid Mechanics 43, 247-272.

De Giorgi, M. G., C. G. De Luca, A. Ficarella and F. Marra (2015). Comparison between synthetic jets and continuous jets for active flow control: Application on a NACA 0015 and a compressor stator cascade. Aerospace Science and Technology 43, 256-280.

Duvigneau, R. and M. Visonneau (2006). Optimization of a synthetic jet actuator for aerodynamic stall control. Computers and Fluids 35(6), 624-638.

Genc, M. S., Ü. Kaynak and H. Yapici (2011). Performance of transition model for predicting low Re aerofoil flows without/with single and simultaneous blowing and suction. European Journal of Mechanics, B/Fluids 30(2), 218235.

Gilarranz, J. L., L. W. Traub and O. K. Rediniotis (2005a). A new class of synthetic jet actuators - Part I: Design, fabrication and bench top characterization. Journal of Fluids Engineering, Transactions of the ASME 127(2), 367-376.

Gilarranz, J. L., L. W. Traub and O. K. Rediniotis (2005b). A new class of synthetic jet actuators - Part II: Application to flow separation control. Journal of Fluids Engineering, Transactions of the ASME 127(2), 377-387.

Godard, G., J. M. Foucaut and M. Stanislas (2006). Control of a decelerating boundary layer. Part 2: Optimization of slotted jets vortex generators. Aerospace Science and Technology 10(5), 394-400.

Godard, G. and M. Stanislas (2006a). Control of a decelerating boundary layer. Part 1: Optimization of passive vortex generators. Aerospace Science and Technology 10(3), 181-191.

Godard, G. and M. Stanislas (2006b). Control of a decelerating boundary layer. Part 3: Optimization of round jets vortex generators. Aerospace Science and Technology 10(6), 455-464.

He, C., T. C. Corke and M. P. Patel (2009). Plasma flaps and slats: An application of weakly ionized plasma actuators. Journal of Aircraft $46(3), 864-873$.

Huang, L., P. G. Huang, R. P. LeBeau and T. Hauser (2004). Numerical study of blowing and suction control mechanism on NACA0012 airfoil. Journal of Aircraft 41(5), 1005-1013.

Kim, S. H. and C. Kim (2009). Separation control on NACA23012 using synthetic jet. Aerospace Science and Technology 13(4-5), 172-182.

Mohamed, G. E. H. (2001, jun). Flow control: passive, active, and reactive flow management. Choice Reviews Online 38(10), 38- 5597-385597.

Müller-Vahl, H. F., C. Strangfeld, C. N. Nayeri, C. O. Paschereit and D. Greenblatt (2015). Control of thick airfoil, deep dynamic stall using steady blowing. AIAA Journal 53(2), 277-295. 
F. Sonkaya et al. / JAFM, Vol. 15, No. 2, pp. 427-440, 2022.

Schmidt, S. and F. Thiele (2003). Detached Eddy Simulation of Flow Around A-Airfoil. Flow, Turbulence and Combustion 71(1-4), 261278.

Seifert, A., T. Bachar, D. Koss, M. Shepshelovich and I. Wygnanski (1993). Oscillatory blowing: A tool to delay boundary-layer separation. AIAA Journal 31(11), 2052- 2060.

You, D. and P. Moin (2009). Active control of flow separation over an airfoil using synthetic jets. Solid Mechanics and its Applications 14, 551561.
Zhao, G. and Q. Zhao (2014). Parametric analyses for synthetic jet control on separation and stall over rotor airfoil. Chinese Journal of Aeronautics 27(5), 1051-1061.

Zheng, J., Y. D. Cui, Z. Zhao, J. M. Li and B. C. Khoo (2018). Flow separation control over a NACA 0015 airfoil using nanosecondpulsed plasma actuator. AIAA Journal 56(6), 22202234. 